\title{
Shape Transformations of Membrane Vesicles from Amphiphilic Triblock Copolymers: A Dissipative Particle Dynamics Simulation Study
}

\section{Xuejin Li}

CAS Key Laboratory of Soft Matter Chemistry, Department of Polymer Science and Engineering, University of Science and Technology of China, Hefei, Anhui 230026, P. R. China

\section{Igor V. Pivkin}

Department of Materials Science and Engineering, Massachusetts Institute of Technology, Cambridge, Massachusetts 02139

\author{
Haojun Liang* \\ CAS Key Laboratory of Soft Matter Chemistry, Department of Polymer Science and Engineering and \\ Hefei National Laboratory for Physical Sciences at Microscale, University of Science and Technology \\ of China, Hefei, Anhui 230026, P. R. China
}

\section{George Em Karniadakis*}

Division of Applied Mathematics, Brown University, Providence, Rhode Island 02912

Received January 15, 2009; Revised Manuscript Received March 11, 2009

\begin{abstract}
Dissipative particle dynamics (DPD) simulations are employed to study the shape transformations of vesicles formed from amphiphilic triblock copolymers. The amphiphilic molecule is built from two different hydrophilic blocks on the sides and a hydrophobic block in the middle. To model the asymmetric membrane in the vesicle, spontaneous curvature is introduced by the difference in repulsive parameters between the two different hydrophilic blocks of the amphiphilic molecule. A plethora of complex vesicle shapes is revealed by the DPD simulations, including some shapes that have not been reported in other simulation studies on vesicles, such as starfish-shaped, toroidal, long rodlike, and inverted vesicles. These simulated vesicles agree with theoretically derived vesicle shapes based on the spontaneous curvature model and also with experimental observations.
\end{abstract}

\section{Introduction}

Block copolymers can self-assemble into a variety of ordered structures in solutions because of the repulsive interactions between the different blocks and the topological constraints caused by the subchains being linked permanently. Amphiphilic block copolymers, which contain hydrophilic groups and hydrophobic groups, represent an interesting class of polymeric materials that exhibit a rich variety of morphologies, ${ }^{1,2}$ such as micelles, membranes, and vesicles, making them a subject of great interest for experiments, theories, and computer simulations. Among these complex microstructures, the vesicles have received increasing attention due to their great potential applications in the fields of drug delivery and nanotechnology.

It is well-known that vesicles are highly adaptive structures having a rich variety of shapes. The study of shape and topological changes has important physical and biological significance. In the past decade, vesicles attracted considerable attention in both experimental and theoretical studies. ${ }^{3-10}$ It was recognized early on that vesicle shapes reflect to a large extent the bilayer nature of amphiphilic membranes. ${ }^{11,12}$ An interesting laboratory study of shape deformations of membrane vesicles caused by temperature changes was performed by Sackmann's group. ${ }^{13,14}$ Further analysis revealed some general features of vesicle shapes that depend on the layered membrane structure. ${ }^{5}$ These experimental results confirm the hypothesis that the shape of a vesicle is mainly determined by bending elasticity and thus

*Corresponding authors. E-mail: gk@dam.brown.edu and hjliang@ ustc.edu.cn. by curvature. A number of theoretical continuum based models explaining the variety of shapes exist. Among them are the spontaneous curvature model (SCM) ${ }^{15,16}$ the bilayer coupling model (BCM) ${ }^{16,17}$ and the area-difference model (ADE). ${ }^{18,19}$ The models and their variants allow the prediction of energyminimizing shapes for a given parameter set. However, despite the large amount of published works on the vesicle shapes in experiment and theory, papers on numerical simulations of vesicle shapes have seldom been reported. Morikawa et al. ${ }^{20}$ studied the vesicle shapes by the Monte Carlo simulation of a joint-segment model. The shape transitions of fluid vesicles and red blood cells in shear flow were studied by Noguchi ${ }^{21,22}$ and Pivkin and Karniadakis ${ }^{23}$ using simulation techniques that combine a three-dimensional particle-based mesoscopic model for the solvent with a triangulated surface model for the membrane. Also, recently the shape transformations of twocomponent lipid vesicles from coarse-grained molecular dynamics (CGMD) simulations have been investigated by Markvoort and co-workers. ${ }^{24}$ However, to the best of our knowledge, no studies of shape transformations of single-component polymer vesicles using the dissipative particle dynamics (DPD) method have been reported.

In this paper, we study the shape transformations of polymer vesicles composed of amphiphilic triblock copolymers using the DPD approach. We show that for a membrane consisting of amphiphilic triblock copolymers, a difference in DPD parameters between two different hydrophilic blocks in amphiphilic molecules can introduce an asymmetry in the membrane, resulting in a spontaneous curvature. In case the 
membrane forms a vesicle, the spontaneous curvature can influence the shapes of the membrane vesicles. We can obtain a wide variety of vesicle shapes by properly adjusting the difference in the repulsive parameters of the DPD system. Both familiar and new shapes are formed, and here we compare our results with theoretically derived shapes and experimental observations whenever possible.

\section{Simulation Details}

2.1. DPD Formulation. Dissipative particle dynamics is a mesoscopic Lagrangian simulation method, introduced in 1992 by Hoogerbrugge and Koelman. ${ }^{25}$ In DPD simulation, a particle represents the center of mass of a cluster of atoms, and the mass, length, and time scales are all unity. Particles $i$ and $j$ interact with each other via a pairwise additive force, consisting of three components: (i) a conservative force, $F_{i j}^{\mathrm{C}}$; (ii) a dissipative force, $F_{i j}^{\mathrm{D}}$; and (iii) a random force, $F_{i j}^{\mathrm{R}}$. All forces are nonzero within a cutoff radius $r_{\mathrm{c}}$. Hence, the total force on particle $i$ is given by

$$
F_{i}=\sum_{i \neq j} F_{i j}^{\mathrm{C}}+F_{i j}^{\mathrm{D}}+F_{i j}^{\mathrm{R}}
$$

where the sum acts over all particles within $r_{\mathrm{c}}$. Specifically, in our simulations

$$
F_{i}=\sum_{i \neq j} a_{i j} \omega\left(r_{i j}\right) \hat{r}_{i j}-\gamma \omega^{2}\left(r_{i j}\right)\left(\hat{r}_{i j} \cdot v_{i j}\right) \hat{r}_{i j}+\sigma \omega\left(r_{i j}\right) \xi_{i j} \Delta t^{-1 / 2} \hat{r}_{i j}
$$

where $a_{i j}$ is a maximum repulsion between particles $i$ and $j, r_{i j}$ is the distance between them, with the corresponding unit vector $\hat{r}_{i j}, v_{i j}$ is the difference between the two velocities, $\zeta_{i j}$ is a random number with zero mean and unit variance, and $\gamma$ and $\sigma$ are parameters coupled by $\sigma^{2}=2 \gamma k_{\mathrm{B}} T$. The weight function $\omega\left(r_{i j}\right)$ is given by

$$
\omega\left(r_{i j}\right)= \begin{cases}1-r_{i j} / r_{\mathrm{c}} & r_{i j}<r_{\mathrm{c}} \\ 0 & r_{i j} \geq r_{\mathrm{c}}\end{cases}
$$

The value $\sigma=2.4$ and $\gamma=4.5$ are used to study the shape transformations of membrane vesicles; therefore, the temperature constant is kept at $k_{\mathrm{B}} T=0.64$. By joining consecutively particles with a spring force, we can construct coarse-grained models of polymers. ${ }^{26,27}$ The harmonic spring force with a spring constant $k_{\mathrm{s}}=10.0$ and an equilibrium bond length $r_{\mathrm{s}}=0.86$ in our simulations has the form

$$
F_{i j}^{\mathrm{S}}=k_{\mathrm{s}}\left(1-r_{i j} / r_{s}\right) \hat{r}_{i j}
$$

The simulations are performed using a modified version of the DPD code named MYDPD. ${ }^{28,29}$ The time integration of motion equations is done using a modified velocity-Verlet algorithm with $\lambda=0.65$ and time step $\Delta t=0.04$; a temporal resolution study is presented in section 4.1.

2.2. DPD Models. Within the mesoscopic approach, an amphiphilic molecule is represented by a coarse-grained model. In order to simulate polymer-based asymmetric membranes as well as vesicles in solvent, we consider the amphiphilic triblock copolymer molecules. For the sake of simplicity, each amphiphilic molecule is built by pairs of hydrophilic particles (denoted by $\mathrm{A} / \mathrm{C}$ ) on each side and six hydrophobic particles (denoted by $\mathrm{B}$ ) in the middle; i.e., an amphiphilic $\mathrm{A}_{2} \mathrm{~B}_{6} \mathrm{C}_{2}$ triblock copolymer molecule is modeled, as shown in Figure 1a. Solvent particles (denoted by $\mathrm{S}$ ) are included explicitly in the simulations; however, for clarity, they are not shown in the following figures.

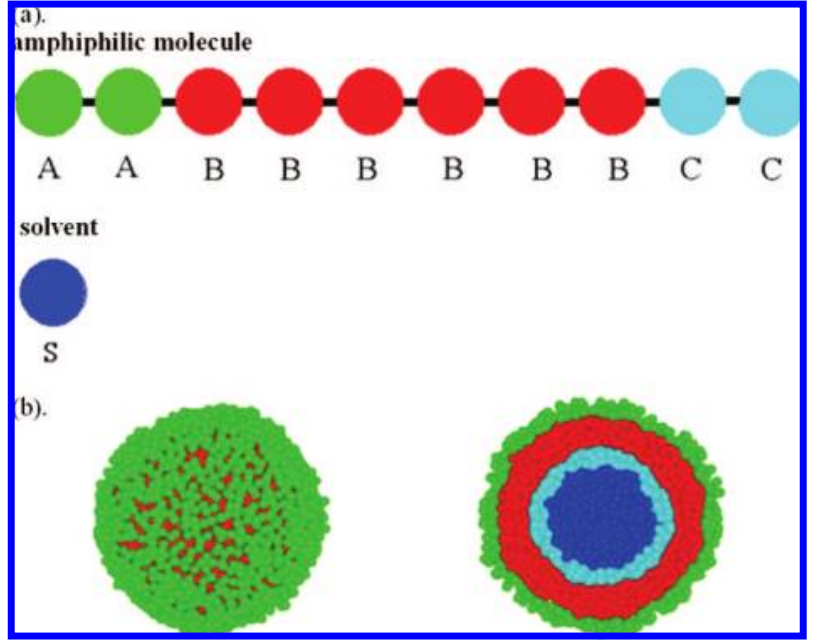

Figure 1. (a) DPD model of $\mathrm{A}_{2} \mathrm{~B}_{6} \mathrm{C}_{2}$ amphiphilic molecule and (b) the self-assembled vesicle obtained in DPD simulations. In the figure, the $\mathrm{A}$ and $\mathrm{C}$ particles are hydrophilic while the $\mathrm{B}$ particles are hydrophobic.

For single-component vesicles consisting of amphiphilic triblock copolymers, the spontaneous curvature of the membrane can be controlled by changing the conservative (repulsive) parameters between particles. Following Laradji and Kumar, ${ }^{30,31}$ the repulsive parameter between two alike particles is set to $a_{i j}$ $=25.0$. The values of the parameters between two particles of which one is hydrophilic and the other hydrophobic are $a_{\mathrm{AB}}=$ $a_{\mathrm{BC}}=200.0$, which ensures that the hydrophobic block of amphiphile is sufficiently shielded from the hydrophilic blocks. For the interaction between two distinct types of hydrophilic particles, we have chosen the value $a_{\mathrm{AC}}=100.0$ such that the two types of hydrophilic particles are in the strong segregation regime. To introduce a spontaneous curvature in the membrane, the repulsive parameter between A particles is chosen to be larger than between $\mathrm{C}$ particles. Specifically, their values are set to $a_{\mathrm{AA}}=30.0$ and $a_{\mathrm{CC}}=20.0$. In summary, the repulsive interaction parameters between any two particles are given by

$$
a_{i j}=\left(\begin{array}{lllll} 
& \mathrm{A} & \mathrm{B} & \mathrm{C} & \mathrm{S} \\
\mathrm{A} & 30.0 & 200.0 & 100.0 & 25.0 \\
\mathrm{~B} & 200.0 & 25.0 & 200.0 & 200.0 \\
\mathrm{C} & 100.0 & 200.0 & 20.0 & 25.0 \\
\mathrm{~S} & 25.0 & 200.0 & 25.0 & 25.0
\end{array}\right)
$$

With these parameters, an asymmetric membrane vesicle with the outer part of the membrane consisting of hydrophilic particles $\mathrm{A}$ and inner part consisting of hydrophilic particles $\mathrm{C}$ can be formed. The area expansion modulus, $K_{\mathrm{A}}$, of the membrane is calculated to be $23.4 k_{\mathrm{B}} T / r_{\mathrm{c}}{ }^{2}$, and the membrane thickness $l_{\mathrm{me}}$ is estimated to be $3.7 r_{\mathrm{c}}$; then the bending rigidity is calculated via $\kappa=K_{\mathrm{A}} l_{\mathrm{me}}{ }^{2} / 48$, which is about $6.8 k_{\mathrm{B}} T$. The value of $\kappa$ in our model is in reasonable agreement with the experimental values for membranes.

A difference in repulsive interactions between two different hydrophilic blocks in amphiphilic molecules introduces an asymmetry in the membrane, resulting in a spontaneous curvature. In case the membrane forms a vesicle, the spontaneous curvature of the membrane will influence the shapes of the vesicle. Therefore, the shape transformations of vesicles can be imposed by changing the repulsive interaction differences between the parameters of the hydrophilic particles $\mathrm{A}$ in the outer part and hydrophilic particles $\mathrm{C}$ in the inner part of the membrane. 


\section{Shape Transformations of Membrane Vesicles}

In this section, we present results from simulations of the $\mathrm{A}_{2} \mathrm{~B}_{6} \mathrm{C}_{2}$ amphiphilic triblock copolymer systems. The simulations are started from a spherical vesicle that is formed from 960 amphiphilic molecules (or 9600 particles) of $\mathrm{A}_{2} \mathrm{~B}_{6} \mathrm{C}_{2}$ amphiphilic triblock copolymers in a simulation box of $40 \times$ $40 \times 40$ with a particle number density of 3 at two different temperatures. At $k_{\mathrm{B}} T=1.00$, we find only a little difference in the repulsive parameters can bring a large shape deformation of vesicles. So, we adopt a strategy that the shape transformations of vesicles are imposed by varying the difference of repulsive interactions in a larger range at a lower temperature $k_{\mathrm{B}} T=0.64$. A rough but simple estimation of the value of this reduced temperature in terms of physical temperature can be given by the following equation: ${ }^{32}$

$$
T=133 T^{*}+240
$$

where $T$ and $T^{*}$ represent the physical temperature and reduced temperature, respectively. We then find $T=325 \mathrm{~K}$ for $k_{\mathrm{B}} T=$ 0.64. The vesicle, which is shown in Figure 1b, contains 3190 solvent particles in the interior. As described in previous simulations based on DPD model, ${ }^{31}$ the large repulsive interaction parameters of the model are selected so that the membrane vesicle is impermeable to solvent particles, which implies that the number of solvent particles inside the closed vesicle is constant. It is hard to change the shape for a spherical vesicle without changing the interior volume. Therefore, the initial configurations for the following simulations are all derived from a vesicle for which a certain number of solvent particles are removed from its interior and placed into the outer region. The shape transformations of vesicles can be imposed by varying the difference of repulsive interactions, $\Delta a=a_{\mathrm{AA}}-a_{\mathrm{CC}}$, in the range from -10.0 to 49.5 by changing the repulsive parameters of $a_{\mathrm{AA}}$ in the range from 20.0 to $49.5 ; a_{\mathrm{CC}}$ is obtained through the relation $a_{\mathrm{CC}}=50.0-a_{\mathrm{AA}}$. A typical simulation requires 500000 time steps, of which 50000 time steps are needed for equilibration.

A plethora of vesicle shapes are observed in the simulations. A selection of vesicle shapes induced by the differences of the repulsive interactions is displayed in Figure 2. The spherical vesicle deforms to achieve oblate/prolate shapes depending on the value of $\Delta a$ just after a certain number of the interior solvent particles are removed. If we continue changing $\Delta a$ for these two shapes, the range of shapes obtained for the membrane vesicle grows larger, and new shapes appear. At low $\Delta a$, the oblate shape is formed from the spherical vesicle. With the decrease of the value of $\Delta a$, it transforms to stomatocyte shape, which is abundant in nature. ${ }^{16,33}$ Further decrease in $\Delta a$ to even smaller (or negative) value causes the oblate to split from the edge and to form an asymmetric monolayer and later to transform into an inverted vesicle. As $\Delta a$ is increased, the oblate shape is transformed continuously into the prolate one via an ellipsoidal shape, which indicates that the prolate shape is favored at higher positive $\Delta a$. Further increase in $\Delta a$ causes formation of branches, resulting in dumbbell and pear-shaped vesicles. These finally transform to fission-produced vesicles through a necklike early intermediate and a hemifission late intermediate. When more solvent particles are removed from the interior of the vesicle to the outer region, we find several novel shapes, such as starfish and toroidal vesicles, which have been studied previously experimentally and theoretically. ${ }^{34-39}$ At still lower $\Delta a$, when more interior solvent particles are removed, the oblate becomes flat and pinches off at the center to form a toroidal vesicle with a single hole (a torus). ${ }^{34,35,40}$ As the $\Delta a$ is increased, the oblate shape is no longer transformed into ellipsoidal or prolate shape; instead, it transforms continu-

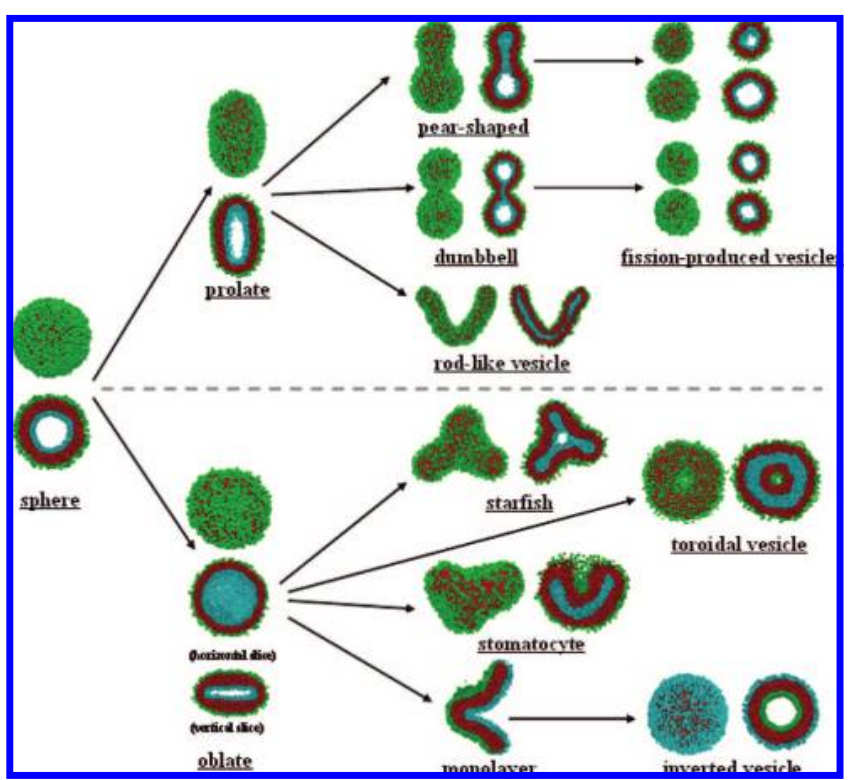

Figure 2. Shape transformation pathways of membrane vesicles from amphiphilic triblock copolymer in DPD simulations. The slices of these shapes are also shown here for clarity. In the upper half, the difference of repulsive interactions, $\Delta a$, has larger positive value whereas in the lower half, $\Delta a$ has smaller or negative value.

ously into a three-armed starfish ${ }^{36-39}$ via an elongated rounded triangular shape.

In order to provide a more quantitative insight into the characteristics of the shape transformation behavior of membrane vesicles, we employ existing theoretical models for comparison. For the three continuum models, the bilayer coupling model and the area-difference model apply to a membrane consisting of two monolayers; however, the amphiphilic membrane in our simulations is essentially a single monolayer. Moreover, the amphiphilic membrane consists of an asymmetric monolayer, and hence it has nonzero spontaneous curvature. Thus, we hypothesize here that the spontaneous curvature model might be the most appropriate model for this membrane. We have also made comparisons with the bilayer coupling model; these results-not shown here-support our hypothesis. In the following sections, we compare the simulated vesicles with the spontaneous curvature model.

\section{Comparison with Spontaneous Curvature Model}

For the spontaneous curvature model, the phase diagram depends only on two dimensionless variables: the reduced spontaneous curvature, $c_{0}$, and the reduced volume, $v$, defined by

$$
v=\frac{V}{4 \pi / 3 R_{0}^{3}}
$$

where $R_{0}$ is the radius of a sphere with the same area. To describe the shape transformations of simulated vesicles in terms of the spontaneous curvature model, the triangulated surface model is used to estimate the volume of the vesicles. Since a relatively small vesicle is used, the thickness of the membrane is not negligible and should be taken into account when estimating its volume. The density of particles forming the vesicle membrane is computed on a three-dimensional grid with a series of nodes. The density isosurfaces can be represented using the triangular surface meshes connecting the corresponding nodes. The membrane formed from amphiphilic molecules has two different hydrophilic domains and two different isosurfaces (where the density is midway between the hydrophilic and 


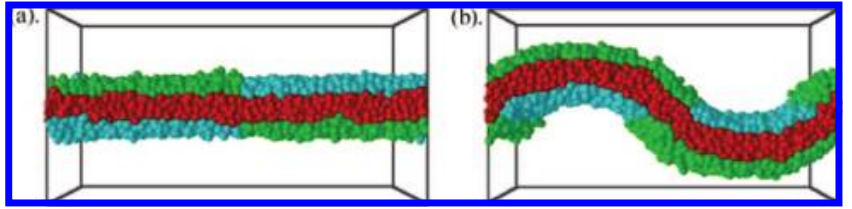

Figure 3. Spontaneous curvature in the resulting monolayer with two opposite domains for (a) $\Delta a=0.0$ and (b) $\Delta a=5.0$.

hydrophobic domains) exist in the membrane, i.e., the inner isosurface between hydrophilic A and hydrophobic B domains and the outer one between hydrophilic $\mathrm{C}$ and hydrophobic $\mathrm{B}$ domains. The volume of the vesicle is calculated by measuring how many vertices of a dense regular grid happen to be within the middle surface of the monolayer, which is located in the middle between the inner and outer isosurfaces. The values of reduced volumes are listed in Table 1 . For a spherical vesicle, we obtain a reduced volume of $\sim 1.0$. A reduced volume of $\sim 0.80$ can be obtained after $40 \%$ of the interior solvent particles are removed from the interior of the vesicle. Similarly, if $80 \%$ of the interior solvent particles are removed, we obtain a vesicle with a reduced volume of $\sim 0.55$.

A simple estimate can be given here. The original vesicle has a radius $\left(R_{0}\right)$ of $8.0 r_{\mathrm{c}}$ and thus a volume $V_{0}=(4 / 3) \pi R_{0}{ }^{3}=2145 r_{\mathrm{c}}{ }^{3}$. The membrane thickness $l_{\mathrm{me}}$ is estimated to be $3.7 r_{\mathrm{c}}$, and the inner radius of the vesicle is $6.2 r_{\mathrm{c}}$ with a volume $V_{\mathrm{in}}=998 r_{\mathrm{c}}{ }^{3}$. If we assume that the membrane volume is constant, a reduced volume $v \approx\left(V_{0}-V_{\text {in }} \times 0.40\right) / V_{0}=0.81$ is obtained by removing $40 \%$ of the interior solvent. When $80 \%$ of interior solvent is removed, the volume ratio drops to $v \approx\left(V_{0}-V_{\text {in }} \times 0.80\right) / V_{0}=0.63$. We noticed that when a certain number of solvent particles are removed from the interior of the vesicle and placed into its outer region, the membrane thickness will increase slightly. This affects the volume of the vesicle. Thus, the calculated reduced volumes decrease faster than predicted by simple estimates presented above. For the purpose of comparison with spontaneous curvature model, we also need to find the relation between the reduced spontaneous curvature $c_{0}$ and the difference of repulsive interactions $\Delta a$ in the simulations.

4.1. Spontaneous Curvature in Membrane. Following Markvoort et al., ${ }^{24}$ a membrane with two large domains consisting of $\mathrm{A}_{2} \mathrm{~B}_{6} \mathrm{C}_{2}$ amphiphilic molecules is simulated in a periodic box to obtain spontaneous curvature. In the simulations, the membrane is oriented perpendicular to the $x$-axis and the area of the $y z$-plane is changed in order to control the membrane tension to be a constant with the given value. This is done by manually changing the membrane projected area on the $y z$-plane and the height of the simulation box to ensure that the total volume of the system remains constant, and therefore no work is done against the external pressure. The structure of the membrane is shown in Figure 3a; in the left half of the membrane, the lower part consists of hydrophilic particles $\mathrm{C}$ while the upper part consists of hydrophilic particles A; in the right half of the membrane it is exactly the other way around. Since two domains with different hydrophilic particles coexist, when we change the difference of repulsive interactions between two different hydrophilic particles from the tensionless state, two domains with opposite curvature are formed. A resulting highly curved membrane in the case of $\Delta a=5.0$ is shown in Figure $3 \mathrm{~b}$. To determine the relation between the changes in our DPD repulsive parameters and the spontaneous curvature of the membrane, we compare the curves obtained from the DPD simulations with predictions for membrane shapes from a spontaneous curvature model that

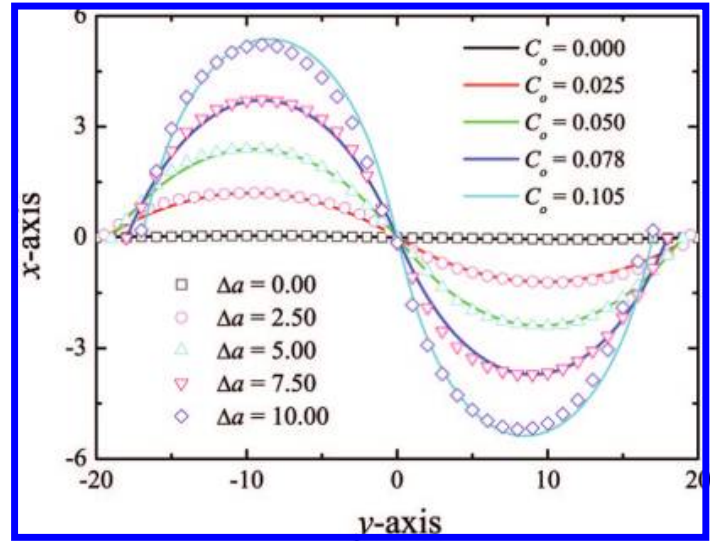

Figure 4. Responses obtained through energy minimization according to eq 9 for various values of the spontaneous curvatures $C_{0}$ (solid lines) and changes of the repulsive parameters (symbols) from simulations.

predicts the energy-minimizing shapes for a given parameter set. In the spontaneous curvature model of Herfrich, ${ }^{15}$ the membrane has bending energy which depends quadratically on its local principal curvatures $C_{1}$ and $C_{2}$ as

$$
E_{\mathrm{b}}=\frac{\kappa_{\mathrm{b}}}{2} \int_{A} \mathrm{~d} a\left(C_{1}+C_{2}-C_{0}\right)^{2}+\frac{\kappa_{\mathrm{G}}}{2} \int_{A} \mathrm{~d} a C_{1} C_{2}
$$

where $\kappa_{\mathrm{b}}$ and $\kappa_{\mathrm{G}}$ are respectively the bending rigidity and Gaussian bending rigidity, and $C_{0}$ is a parameter representing the spontaneous curvature that a membrane may possess arising from compositional inhomogeneities in its monolayer. Since curvature in the membrane is expected only in one dimension, the equation reduces to

$$
E_{\mathrm{b}}=\frac{\kappa_{\mathrm{b}} l_{\mathrm{me}}}{2} \int_{0}^{L}\left(C_{1}-C_{0}\right)^{2} \mathrm{~d} s
$$

where $l_{\mathrm{me}}$ is the membrane thickness. The responses obtained in simulations by changing the values of spontaneous curvature through variation of the parameters of repulsive interactions are shown in Figure 4. Several recent works showed that using a time step of 0.04 in simulations of model bilayer membranes may lead to deviations in the distribution of stresses within the membrane that are as large as the physically meaningful quantities found for smaller time steps. ${ }^{41,42}$ In order to investigate possible effects of the time step in simulations of our particular membrane, we repeated all simulations at different time steps. We concluded that our results are independent of the time step in the range we have selected; typical results of this systematic temporal resolution study are shown in Figure 5a,b.

By comparing the curves obtained from theory and simulations, the spontaneous curvature $C_{0}$ can be plotted as a function of the difference in repulsive forces $\Delta a$ between $a_{\mathrm{AA}}$ and $a_{\mathrm{CC}}$, as shown in Figure 6. Then, the functional dependence of the reduced spontaneous curvature $c_{0}$ on the difference of repulsive forces $\Delta a$ is obtained by a linear fitting method, i.e.

$$
c_{0}=C_{0} R_{0}=0.0834 \Delta a+0.0008
$$

Once the reduced spontaneous curvature $c_{0}$ has been determined

\begin{tabular}{|c|c|c|c|c|c|c|c|c|c|}
\hline percentage of interior solvent remov & 0 & 10 & 20 & 30 & 40 & 50 & 60 & 70 & 80 \\
\hline volume $\left(r_{\mathrm{c}}^{3}\right)$ & 2164 & 2061 & 1955 & 1845 & 1731 & 1612 & 1485 & 1346 & 1190 \\
\hline reduced volume & 1.00 & 0.95 & 0.90 & 0.85 & 0.80 & 0.74 & 0.69 & 0.62 & 0.55 \\
\hline
\end{tabular}
for a specific vesicle, other attributes such as the reduced volume

Table 1. Obtained Reduced Volumes of Vesicles by Removing Percents of Solvent from the Interior of the Vesicle to the Outer Region 

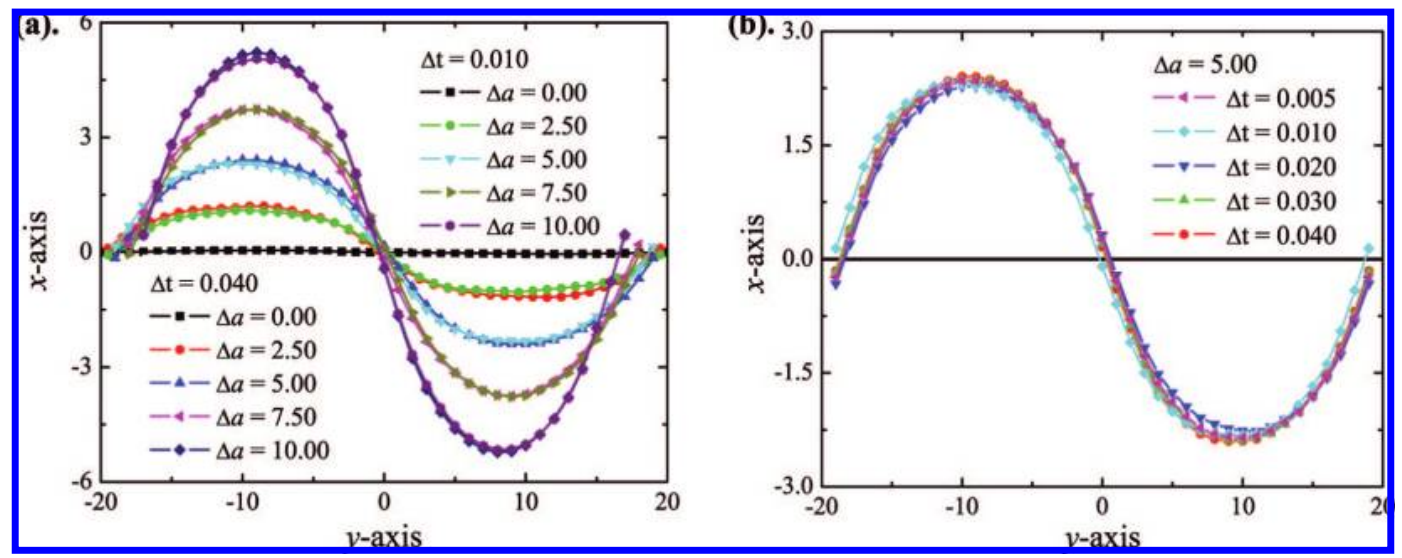

Figure 5. (a) Same plot as in the previous figure but for two different time steps. (b) Time resolution tests for $\Delta a=5.0$ and different values of the time step.

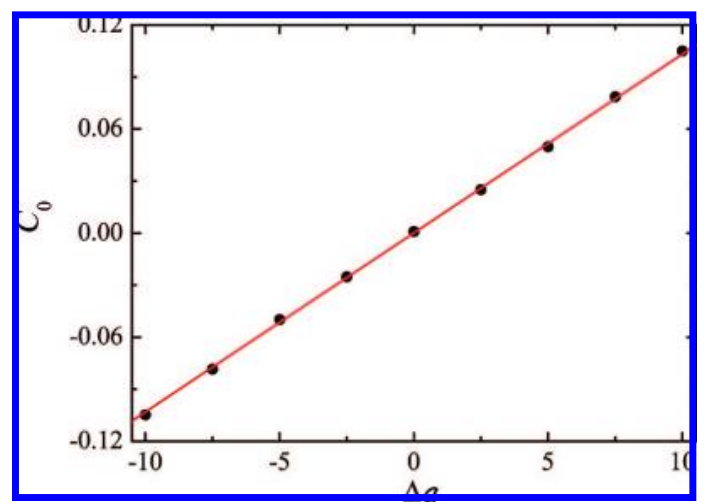

Figure 6. Functional dependence of the spontaneous curvature $C_{0}$ on the difference repulsive forces $\Delta a$ between $a_{\mathrm{AA}}$ and $a_{\mathrm{CC}}$ obtained from simulations.

$v$ of vesicle can be varied, and the resulting change in shapes can be compared against both theoretical prediction and experimental results. Similarly, a comparison in opposite direction can be obtained, i.e., once the reduced volume $v$ has been determined for a specific vesicle, the values obtained for the spontaneous curvature $c_{0}$ can be compared to the theoretical prediction as well as experimental observations.

4.2. Shape Transformations of Membrane Vesicles for Fixed Reduced Volume. Let us consider vesicle shapes of the same reduced volume with different spontaneous curvatures $c_{0}$. Specifically, we chose the value of $v=0.80$, for which the results can be compared to the theoretical prediction by the spontaneous curvature model reported in Markvoort et al. ${ }^{24}$ The variation of vesicle shape as a function of the reduced spontaneous curvature at the fixed volume fraction are shown in Figure 7. In the order from low to high values of reduced spontaneous curvature, inverted-shaped, stomatocyte, oblate, prolate, pearshaped, and fission-produced vesicles are observed. The shapes appear in the same order as predicted by the spontaneous curvature model for $c_{0}>0$; however, for $c_{0} \leq 0.0$, we find similar order with the theoretical prediction ${ }^{24}$ but also new shapes; e.g., for $c_{0}=-1.0$, we obtain the inverted spherical vesicle shape with the outer part of the membrane consisting of hydrophilic particles $\mathrm{C}$ and the inner part consisting of hydrophilic particles A. The reduced volume goes back to 1.00 for the inverted vesicle since it is formed after rupture. The appearance of the inverted vesicle can be explained by the fact that an asymmetry exists in the membrane of the original vesicle. A change in repulsive interactions between two different hydrophilic particles in amphiphilic molecules influences the packing of the amphiphiles in the monolayer, causing a slight

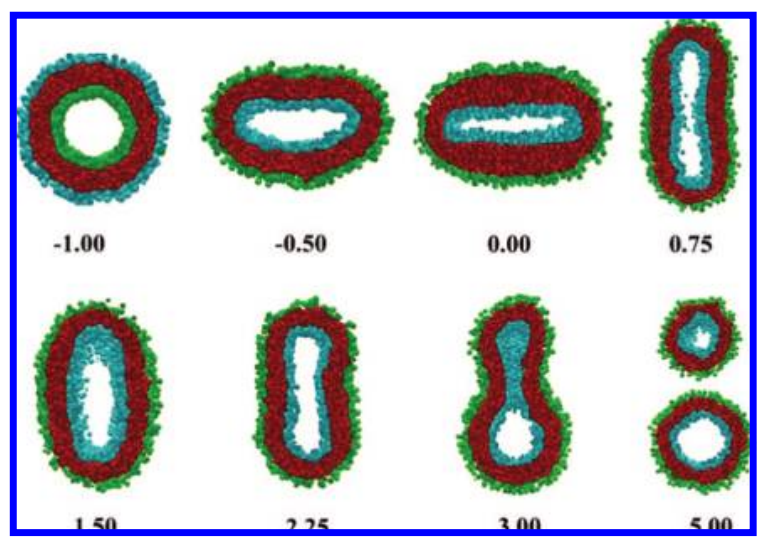

Figure 7. Shape transformations of membrane vesicles as a function of the reduced spontaneous curvature by changing the repulsive parameters between two different hydrophilic particles at the reduced volume $v=0.80$, except for the $c_{0}=-1.00$ (the inverted vesicle formed after rupture makes the reduced volume go back to 1.00).

change of the membrane area. For a relative large negative value of $\Delta a$, the vesicle can rupture and form an inverted vesicle.

4.3. Shape Transformations of Membrane Vesicles for Fixed Reduced Spontaneous Curvature. For the purpose of comparison with the spontaneous curvature model in the case of fixed spontaneous curvature, we chose the same value of spontaneous curvature in our simulations as in Seifert et al. ${ }^{16}$ A series of simulations with fixed repulsive interaction parameters were performed, varying the reduced volume $v$ by removing some amount of solvent particles from the interior of vesicle into the outer region. In Figure 8 some simulated shapes of membrane vesicles are shown with the corresponding values of the reduced volume $v$ for fixed reduced spontaneous curvature $c_{0}=3.0$. The shapes from simulations correspond to pear-shaped and dumbbell classes.

At $v=1.0$, the vesicle shape is always spherical. As $v$ is decreased from unity, the sphere is transformed into a prolate shape, elongating continuously. Upon further decrease of $v$, the prolate is replaced by the pear-shaped vesicle, where a large and a small sphere are connected by a narrow neck. As the reduced volume is reduced to 0.70 , the dumbbell shape appears. A further decrease of $v$ leads to the gradual increase of the neck length, while the shapes remain basically the same. The sequence of shapes found is quantitatively the same as the theoretical prediction by Seifert et al. ${ }^{16}$ However, the multispherical vesicle connected by narrow necks, which is predicted by theory, does not appear in our simulations. The reason for 


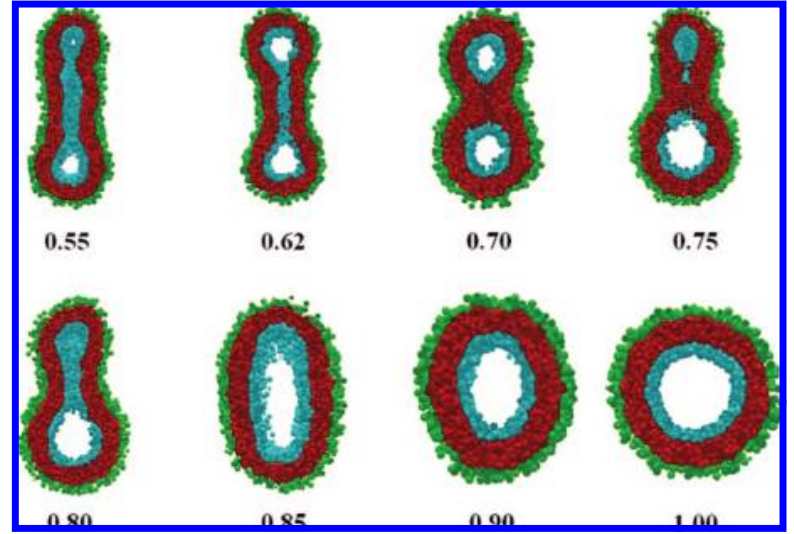

Figure 8. Shape transformations of membrane vesicles as a function of the reduced volume $v$ at the reduced spontaneous curvature $c_{0}=$ 3.0.

this might be the size of the vesicle in our simulations, which is relatively small.

\section{Conclusion}

In this paper, we studied shape transformations of vesicles by particle-based models of amphiphilic triblock copolymers for the first time using the dissipative particle dynamics (DPD) approach. The DPD model of an amphiphilic molecule has two different hydrophilic blocks in each side. The amphiphilic membrane is essentially a single monolayer with the amphiphilic molecule responsible for the hydrophobic core as well as both hydrophilic ends. This is different from the work of Markvoort et al., ${ }^{24}$ who used coarse-grained molecular dynamics (CGMD) models of lipid bilayer membranes. The spontaneous curvature of the membrane is controlled by the difference in repulsive parameters between the hydrophilic blocks of the amphiphilic molecules. A plethora of complex vesicle shapes, including some that have not been reported in previous simulation studies on vesicles, such as starfish-shaped, toroidal, long rod-like and inverted vesicles, are obtained in the DPD simulations. The results are in agreement with experimental observations and theoretical predictions. These findings demonstrate that DPD method is an effective (relatively simple) simulation technique for understanding the behavior of amphiphilic block copolymer vesicles.

Acknowledgment. We thank the three anonymous referees whose critical comments helped us in improving the quality of our manuscript. We are grateful for the financial support provided by the Outstanding Youth Fund (No. 20525416) and the Program of the National Natural Science Foundation of China (Nos. 20874094 and 50773072), NBRPC (No. 2005CB623800). G. Karniadakis acknowledges the financial support provided by the NSF/IMAG program. X. Li gratefully acknowledges the hospitality of Prof. G.
Karniadakis and Brown University, where the work was carried out when he was a visiting researcher. Simulations were carried out at the Shanghai Supercomputer Center.

\section{References and Notes}

(1) Bates, F. S.; Fredrickson, G. H. Phys. Today 1999, 52, 32.

(2) Alexandridis, P.; Lindman, B. Amphiphilic Block Copolymers: SelfAssembly and Applications; Elsevier: New York, 2000.

(3) Lipowsky, R. Nature (London) 1991, 349, 475.

(4) Dobereiner, H. G.; Evans, E.; Kraus, M.; Seifert, U.; Wortis, M. Phys. Rev. E 1997, 55, 4458.

(5) Seifert, U. Adv. Phys. 1997, 46, 13.

(6) Baumgart, T.; Hess, S. T.; Webb, W. W. Nature (London) 2003, 425, 821.

(7) Zhou, Y.; Yan, D. Angew. Chem., Int. Ed. 2004, 43, 4896.

(8) Mecke, A.; Dittrich, C.; Meier, W. Soft Matter 2006, 2, 751.

(9) Kantsler, V.; Segre, E.; Steinberg, V. Phvs. Rev. Lett. 2007, 99, 178102.

(10) Abkarian, M.; Viallat, A. Soft Matter 2008, 4, 653.

(11) Helfrich, W. Z. Naturforsch. 1974, $29 c, 510$.

(12) Evans, E. A. Biophys. J. 1974, 14, 923.

(13) Berndl, K.; Kas, J.; Lipowsky, R.; Sackmann, E.; Seifert, U. Europhys. Lett. 1990, 13, 659.

(14) Kas, J.; Sackmann, E. Biophys. J. 1991, 60, 825.

(15) Helfrich, W. Z. Naturforsch. 1973, 28c, 693.

(16) Seifert, U.; Berndl, K.; Lipowsky, R. Phys. Rev. A 1991, 44, 1182.

(17) Svetina, S.; Zeks, B. Eur. Biophys. J. 1989, 17, 101.

(18) Heinrich, V.; Svetina, S.; Zeks, B. Phys. Rev. E 1993, 48, 3112.

(19) Miao, L.; Seifert, U.; Wortis, M.; Dobereiner, H. G. Phys. Rev. E 1994, 49, 5389.

(20) Morikawa, R.; Saito, Y.; Hyuga, H. J. Phys. Soc. Jpn. 1997, 66, 2513.

(21) Noguchi, H.; Gompper, G. Proc. Natl. Acad. Sci. U.S.A. 2005, 102 , 14159.

(22) Noguchi, H.; Gompper, G. Phys. Rev. E 2005, 72, 011901.

(23) Pivkin, I. V.; Karniadakis, G. E. Phvs. Rev. Lett. 2008, 101, 118105.

(24) Markvoort, A. J.; van Santen, R. A.; Hilbers, P. A. J. J. Phvs. Chem. $B$ 2006, 110, 22780.

(25) Hoogerbrugge, P. J.; Koelman, J. M. V. A. Europhys. Lett. 1992, 19, 155.

(26) Groot, R. D.; Warren, P. B. J. Chem. Phys. 1997, 107, 4423.

(27) Groot, R. D.; Madden, T. J. J. Chem. Phys. 1998, 108, 8713.

(28) De Fabritiis, G.; Serrano, M.; Espanol, P.; Coveney, P. V. Physica A 2006, 361, 429.

(29) Serrano, M.; De Fabritiis, G.; Espanol, P.; Coveney, P. V. Math. Comput. Simul. 2006, 72, 190.

(30) Laradji, M.; Kumar, P. B. S. Phvs. Rev. Lett. 2004, 93, 198105.

(31) Laradji, M.; Kumar, P. B. S. J. Chem. Phys. 2005, 123, 224902.

(32) Venturoli, M.; Smit, B.; Sperotto, M. M. Biophys. J. 2005, 88, 1778.

(33) Lim, G.; Wortis, M.; Mukhopadhyay, R. Proc. Natl. Acad. Sci. U.S.A. 2002, 99, 16766.

(34) Seifert, U. Phys. Rev. Lett. 1991, 66, 2404.

(35) Du, Q.; Liu, C.; Wang, X. Q. J. Comput. Phvs. 2006, 212, 757.

(36) Wintz, W.; Dobereiner, H. G.; Seifert, U. Europhys. Lett. 1996, 33, 403.

(37) Ziherl, P.; Svetina, S. Europhvs. Lett. 2005, 70, 690.

(38) Michalet, X. Phys. Rev. E 2007, 76, 021914.

(39) Yanagisawa, M.; Imai, M.; Taniguchi, T. Phvs. Rev. Lett. 2008, 100 , 148102.

(40) Fourcade, B.; Mutz, M.; Bensimon, D. Phys. Rev. Lett. 1992, 68, 2551.

(41) Jakobsen, A. F.; Mouritsen, O. G.; Besold, G. J. Chem. Phys. 2005, 122, 204901.

(42) Allen, M. P. J. Phys. Chem. B 2006, 110, 3823.

MA9000918 